\title{
SELEÇÃO DE MATRIZES E TIPO DE PROPÁGULO NA ENXERTIA DE SUBSTITUIÇÃO DE COPA EM Ilex paraguariensis ${ }^{1}$
}

\author{
Ivar Wendling ${ }^{2}$, Osmir J. Lavoranti², Marcos Deon V. Resende² e Harry A. Horrmann²
}

\begin{abstract}
RESUMO - Objetivou-se avaliar a técnica da sobreenxertia em Ilex paraguariensis, visando a substituição de copas. Para tanto, foi avaliada a influência da posição do enxerto na planta matriz, a idade e a matriz, bem como, a definição da melhor época para avaliação do pegamento e vigor dos enxertos. Árvores com 13 anos de idade localizadas em Colombo - PR, foram submetidas ao corte a $10 \mathrm{~cm}$ do solo para indução de brotações, sobre as quais foi realizada a enxertia por garfagem no topo em fenda cheia. Os enxertos foram coletados de sete árvores matrizes com idade de 10 anos e sete árvores com idade acima de 80 anos. Das matrizes selecionadas foram retirados enxertos observando-se a posição apical, mediana e basal dentro da planta matriz. O experimento foi instalado em delineamento inteiramente casualizado com três repetições e 42 tratamentos, no esquema fatorial hierárquico 2 × 7 × 3 (duas idades; 7 matrizes, para cada idade; e 3 posições de coleta). A variável resposta de interesse foi proporção de pegamentos e número e comprimento de brotos. Obteve-se um comportamento diferenciado das matrizes quando amostradas em diferentes posições de coleta. A herdabilidade individual dos efeitos genotípicos, estimada para o pegamento dos enxertos, foi superior a $74 \%$, para todos os períodos de avaliação, cujo valor é considerado alto. O coeficiente de variação genética foi da ordem de 34\%, para os períodos de acompanhamento iguais ou superiores a 90 dias, indicando boas perspectivas de ganho no melhoramento do material genético. O coeficiente de variação experimental, nesse mesmo período, ficou entre $78 \%$ e $94 \%$. Não houve diferenças entre os parâmetros genéticos a partir dos 90 dias. Pode-se concluir que a sobreenxertia de erva-mate diretamente a campo é tecnicamente viável; melhores taxas de pegamento são obtidas em árvores mais novas e com brotações apicais; e as matrizes apresentam respostas diferenciadas quanto ao pegamento na enxertia.
\end{abstract}

Palavras-chave: Propagação vegetativa, sobreenxertia e erva-mate.

\section{STOCK PLANTS SELECTION AND RAMET TYPES FOR CROWN GRAFTING IN Ilex paraguariensis}

\begin{abstract}
The objective of this research was to evaluate the technique of topgrafting in Ilex paraguariensis. For that reason, the influence of the position of the graft in the stock plant, the age and the stock plant, as well as, the definition of the best time for grafting evaluation and vigor of the grafts were evaluated. 13 years old trees located in Colombo-PR were submitted to the cut at $10 \mathrm{~cm}$ above the soil for sprouts induction, on which the grafting was accomplished by top cleft grafting. The grafts were collected from 10 year-old mother trees and from seven trees older than 80 years. From the selected trees grafts were taken from the top, medium and basal position inside the mother tree. The experiment was installed with three replications and 42 treatments, in a hierarchical factorial design $2 \times 7 \times 3$ (two ages, seven mother trees for each age and three collection positions). The variables of interest were percentage of survival and number and length of sprouts. A differentiated performance of the mother trees when in different collection positions was observed. The individual heritability of the genotipic effects, estimated for the survival of the grafts, was superior to $74 \%$, for all the evaluation periods, which is considered very high. The coefficient of genetic variation was $34 \%$, for the observed periods same or superior to 90 days, indicating good genetic gain perspectives for
\end{abstract}

\footnotetext{
${ }^{1}$ Recebido em 22-11-2007 e aceito para publicação em 23.06.2009.

${ }^{2}$ Empresa Brasileira de Pesquisa Agropecuária. Colombo, PR - Brasil. E-mail: <ivar@ cnpf.embrapa.br>.
} 
the improvement of the genetic material. The coefficients of experimental variation, obtained for that same periods were between $78 \%$ and $94 \%$. It can be observed that there were no differences among the genetic parameters indicating the possibility of evaluation of the survival of the grafts after 90 days. Based on those results it can be concluded that the Ilex paraguariensis topgrafting directly in the field is technically viable; better survival of grafting are obtained for young trees and with apical sprouts; mother trees have shown differentiated responses in respect to survival of grafting.

Keywords: Vegetative propagation, crown grafting and Ilex paraguariensis.

\section{INTRODUÇÃO}

Ilex paraguariensis St. Hill. (Aquifoliaceae), também conhecida como erva-mate, é uma espécie arbórea típica das regiões subtropicais e temperadas da América do Sul, desempenhando papel socioeconômico importante no Brasil para os Estados de Santa Catarina, Rio Grande do Sul, Paraná e Mato Grosso do Sul. É utilizada na produção de bebidas, principalmente o mate, ou chimarrão, mas apresenta potencial para outras aplicações na indústria como corantes, conservantes alimentares, medicamentos, produtos de higiene e cosméticos (MACCARI e MAZUCHOWSKI, 2000). Dentre as propriedades medicinais, além de ser estimulante, apresenta ação diurética, estomáquica e sudorífica (BRAGAGNOLO et al., 1980).

A produção de mudas de erva-mate via sementes apresenta uma série de limitações, podendo-se destacar: dormência das sementes; longo tempo destinado à estratificação (de quatro a seis meses); germinação demorada, desuniforme e em baixo percentual (de 5 a 20\%) (STURION, 1988); longo período de produção de mudas em viveiro; e dificuldade de obtenção de sementes de boa qualidade. Os plantios de erva-mate provenientes de sementes coletadas sem critérios técnicos apresentam desenvolvimento heterogêneo, com reflexos negativos na produtividade e qualidade do produto final. Esses problemas podem ser minimizados ou até solucionados por meio da obtenção de mudas via propagação vegetativa de indivíduos geneticamente superiores.

A utilização da propagação vegetativa para a produção comercial de mudas de erva-mate, originando indivíduos geneticamente idênticos aos da planta-matriz, pode resolver, pelo menos em parte, essas limitações, resultando na formação de plantios de alta produtividade e uniformidade, possibilitando a produção de mudas durante o ano todo, por meio de plantas-matriz selecionadas (WENDLING e SOUZA JUNIOR, 2003; XAVIER et al., 2001).

R. Árvore, Viçosa-MG, v.33, n.5, p.811-819, 2009
A enxertia tem sido o método mais utilizado na formação de pomares clonais de produção de sementes melhoradas, objetivando à ampliação da base genética após a recombinação entre as matrizes selecionadas, além da multiplicação de fenótipos superiores, formação de pomares de sementes e de bancos clonais (ROCHA et al., 2002).

A sobreenxertia, também conhecida por enxertia de copa ou substituição da parte aérea, é a operação que tem por finalidade o aproveitamento de plantas já formadas com alteração da variedade da copa (PINHEIRO et. al., 1988). O seu emprego mostra-se indicado para plantas de idade não muito avançada e sadias ou para plantas com problemas na parte aérea. Com a sobre-enxertia se ganha tempo, pois o portaenxerto se encontra perfeitamente implantado, e as produções se tornam mais precoces (PINHEIRO et. al., 1988). Para proceder a sobre-enxertia, decepa-se a copa, deixando-se brotar de 4 a 5 ramos, sobre os quais se fará a enxertia da variedade desejada. É o processo indicado para Hevea brasiliensis, quando em plantas adultas, a parte aérea encontra-se atacada por patógenos prejudiciais ao seu desenvolvimento (PINHEIRO et. al., 1988; EMBRAPA, 1989) e poderá vir a ser potencial para a erva-mate, desde que desenvolvida de maneira tecnicamente eficiente.

Torna-se perceptível a falta de estudos mais aprofundados no tocante as técnicas de enxertia em erva-mate. Em se tratando de enxertia diretamente no campo e sobre-enxertia, não se dispõem de resultados. Entretanto, é importante que este processo seja desenvolvido e adaptado, uma vez que poderá resultar em aceleração do processo de substituição de materiais genéticos inadequados já estabelecidos no local de plantio definitivo. Além disso, tem-se a possibilidade de evitar ou diminuir a ocorrência de problemas com enovelamento do sistema radicular em porta-enxertos produzidos em recipientes, entre outros. 
Com base no exposto, este estudo objetivou avaliar para a erva-mate: a) a técnica de sobreenxertia diretamente a campo; b) a influência da posição do enxerto e idade da planta-matriz sobre o pegamento dos enxertos; c) a ocorrência de variações no pegamento dos enxertos entre matrizes e possibilidade de seleção genética para essa característica e; d) a definição da melhor época de avaliação do pegamento de enxertos de erva-mate.

\section{MATERIAL E MÉTODOS}

\subsection{Obtenção dos porta-enxertos}

Árvores com 13 anos de idade localizadas no município de Colombo - PR, foram submetidas ao corte a $10 \mathrm{~cm}$ do solo em agosto de 2001, para indução de brotações. Um ano após foi realizada a enxertia sobre as brotações resultantes.

\subsection{Origem e transporte dos enxertos}

Os enxertos foram coletados de 14 árvores matriz selecionadas com base em seu vigor e resistência a doenças, sendo sete estabelecidas em teste de progênies e procedências em Ponta Grossa - PR, com idade de 10 anos (A1, A2, A3, A7, A8, A9, A10) e sete com idade acima de 80 anos, nativas da região de São Mateus do Sul, PR (F1, F2, F3, F4, F7, F8 e M1). As matrizes receberam podas da parte aérea para colheita de folhas a cada dois anos. Das matrizes selecionadas foram retirados enxertos observando-se a posição dos mesmos dentro da planta matriz, ou seja, apical, mediana e basal. Os enxertos foram transportados em caixas de isopor com gelo recoberto por camadas de papel.

\subsection{Procedimentos de enxertia}

O método de enxertia utilizado foi o de garfagem em fenda cheia, onde o porta-enxerto foi cortado entre 15 e $30 \mathrm{~cm}$ de altura. Posteriormente, foi efetuado um corte de aproximadamente $5 \mathrm{~cm}$ no sentido longitudinal, passando pela medula, onde foi introduzido o enxerto com duas ou três gemas (com tamanho ao redor de $15 \mathrm{~cm}$ ), preparado em cunha e amarrado com fitilho. Ao final da enxertia, envolvendo o enxerto e a região enxertada, foi colocado um saco plástico e sobre este, um saco de papel Kraft (marrom); o primeiro com a finalidade de manter umidade no local, e o segundo visando a proteção do material enxertado da insolação direta.
Em torno de 60 dias após a enxertia, retiraram-se os sacos de papel e foi feita uma abertura dos sacos plásticos, visando à aclimatação, os quais foram completamente retirados aos 70 dias; os fitilhos foram retirados 90 dias após a enxertia. Durante todo o processo, uma vez por semana foi realizada a retirada das brotações dos porta-enxertos.

\subsection{Coleta de dados e análises}

O experimento foi instalado em delineamento inteiramente casualizado com três repetições e 42 tratamentos, no esquema fatorial hierárquico (as matrizes são diferentes em cada classe de idade) 2 × 7 × 3 [(duas idades: acima de 80 anos e 10 anos; 7 matrizes de erva-mate, para cada idade e 3 posições de coleta: A (apical), B (basal) e M (mediana)]. A variável resposta de interesse foi proporção de pegamentos, medida em quatro períodos de acompanhamento (30, 90, 120 e 180 dias), além do número e comprimento de brotos aos 180 dias. A proporção de pegamentos foi estudada como a soma de eventos de Bernoulli independentes (MOOD et al., 1974).

Consideram, para efeito de modelagem estatística, efeitos aleatórios de idade e matrizes e efeito fixo para sobre as posições de coleta. Os diferentes ambientes foram relacionados à origem dos propágulos, o qual foi comum a todas as matrizes. Os efeitos de matrizes e procedências foram referidos como efeitos de genótipos, em que para cada idade foram avaliados sete genótipos distintos. A influência dos efeitos genotípicos na média de cada idade foi obtida pela razão entre a variação genotípica na população e o número de genótipos distintos (RESENDE, 2002). Assim, apenas 1/7 (ou 14\%) da variação genotípica entre os indivíduos ficou retida no efeito da média de idades. Dessa forma, a variação entre as médias de idades foi predominantemente devida às idades propriamente ditas. $\mathrm{O}$ efeito dos porta-enxertos, testado a priori, foi não significativo (valor $\mathrm{p}=0,5642$ ).

As análises de deviance, da proporção de pegamentos, do número e do comprimento de brotos foram executadas pelo Método de Máxima Verossimilhança Restrita-REML (LITTEL et al., 1996). Para a proporção, o melhor ajuste do modelo foi obtido pela distribuição binomial e pela função de ligação Logit. O número de brotos seguiu a distribuição de Poisson e ligação Logarítmica (Log), e o comprimento de brotos foi melhor observado através da distribuição Gamma e função de ligação Log. Para a verificação dos ajustes do modelo, foi utilizado o gráfico meio-normal (half-normal plot)

R. Árvore, Viçosa-MG, v.33, n.5, p.811-819, 2009 
com envelope simulado (COLLETT, 1991). Os componentes de variâncias, parâmetros genéticos e fenotípicos e valores genéticos foram obtidos por meio do programa genético, estatístico "SELEGEN - REML/ BLUP” (RESENDE, 2002), usando-se o modelo 96.

\section{RESULTADOS E DISCUSSÃO}

A análise dos dados, como se as observações fossem independentes, em um esquema fatorial hierárquico, e utilizando-se para a seleção de modelos o Critério Bayesiano de Schwartz (BIC) e o Critério de Informação de Akaike (AIC), encontra-se na Tabela 1. A análise do efeito fatorial cruzado para cada idade independente, não levando em consideração a correlação entre as observações ao longo do tempo, está apresentada na Tabela 2. A análise de deviance, quanto aos caracteres número de brotos e comprimento de brotos aos 180 dias após a enxertia, está apresentada nas Tabelas 3 e 4. As estimativas dos parâmetros genéticos para o pegamento e vigor de enxertos de Ilex paraguariensis estão apresentadas na Tabela 5. Os efeitos genotípicos preditos para o pegamento, comprimento e número de brotos de enxertos de erva-mate (Ilex paraguariensis) estão listados na Tabela 6. Na Tabela 7 é apresentada a média dos efeitos fixos para a posição de coleta de enxertos dentro das plantas-matriz de Ilex paraguariensis em relação ao pegamento, comprimento de brotos e número de brotos dos enxertos.
Observando os dados da Tabela 1, em um esquema fatorial hierárquico e utilizando para a seleção de modelos o Critério Bayesiano de Schwartz (BIC) e o Critério de Informação de Akaike (AIC), pode-se verificar que o desdobramento do modelo para cada idade proporcionou os menores valores para dos referidos critérios, optando-se, assim, pela análise do efeito fatorial cruzado para cada idade independente (Tabela 2).

Aos 30 dias após a enxertia, a sobrevivência dos enxertos foi extremamente alta (média de 93,2\% para as matrizes de 10 anos e $91,1 \%$ para as matrizes acima de 80 anos). No entanto, nas avaliações seguintes esses valores tiveram reduções significativas, demonstrando que a avaliação aos 30 dias não apresenta confiabilidade. Aos 180 dias após a enxertia, observam-se médias de 57,8 e $42,8 \%$, respectivamente, para as matrizes de 10 anos e acima de 80 anos, demonstrando a influência da idade das plantas-matriz e entre estas no pegamento dos enxertos (Tabela 1).

Deve-se notar que o efeito significativo da interação Matriz x Posição de coleta (valor p< 0,0001) e Matriz x Período de acompanhamento, para matrizes com 10 anos (valor $\mathrm{p}=0,0130$ ), supondo a distribuição binomial para a variável resposta proporção de pegamento, confirmou a escolha do modelo pelos critérios AIC e BIC (Tabela 2). Nota-se, também, que a deviance residual foi muito superior ao número de graus de liberdade, mostrando a adequação do modelo utilizado.

Tabela 1 - Médias de pegamento, número de brotos (Nbrotos) e comprimento de brotos (Cbrotos) dos enxertos das 14 matrizes estudadas de Ilex paraguariensis aos 30,90,120 e 180 dias após a enxertia.

Table 1 - Survival averages, number of sprouts (Nbrotos) and length of sprouts (Cbrotos) of the grafts of the 14 studied mother trees of Ilex paraguariensis to the 30, 90, 120 and 180 days after the grafting.

\begin{tabular}{|c|c|c|c|c|c|c|c|c|}
\hline & & & \multicolumn{4}{|c|}{ Pegamento $(\%)$} & \multirow{2}{*}{$\begin{array}{l}\text { Nbrotos } \\
180 \text { dias }\end{array}$} & \multirow{2}{*}{$\begin{array}{l}\text { Cbrotos } \\
180 \text { dias }\end{array}$} \\
\hline & & & 30 dias & 90 dias & 120 dias & 180 dias & & \\
\hline \multirow[t]{16}{*}{ Matrizes } & 10 anos & A 1 & 100,0 & 23,8 & 19,1 & 19,1 & 1,1 & 30,3 \\
\hline & & A 2 & 95,2 & 61,9 & 57,1 & 57,1 & 4,9 & 135,2 \\
\hline & & A 3 & 100,0 & 85,7 & 71,4 & 71,4 & 2,6 & 56,8 \\
\hline & & A7 & 95,2 & 71,4 & 61,9 & 57,1 & 3,3 & 72,5 \\
\hline & & A 8 & 85,7 & 80,9 & 61,9 & 61,9 & 5,9 & 114,5 \\
\hline & & A9 & 100,0 & 71,4 & 66,6 & 66,6 & 2,1 & 31,3 \\
\hline & & A 10 & 80,9 & 76,2 & 71,4 & 71,4 & 3,1 & 77,1 \\
\hline & & Média & 93,2 & 66,0 & 58,5 & 57,8 & 3,3 & 74,0 \\
\hline & $>80$ anos & $\mathrm{F} 1$ & 95,2 & 52,4 & 38,1 & 33,3 & 2,3 & 78,9 \\
\hline & & $\mathrm{F} 2$ & 100,0 & 76,2 & 66,7 & 66,7 & 4,4 & 94,8 \\
\hline & & F3 & 95,2 & 52,4 & 52,4 & 52,4 & 3,5 & 117,1 \\
\hline & & $\mathrm{F} 4$ & 61,9 & 9,5 & 4,8 & 4,8 & 1,3 & 50,0 \\
\hline & & F7 & 85,7 & 57,2 & 52,4 & 52,4 & 3,8 & 101,7 \\
\hline & & F8 & 100,0 & 33,3 & 33,3 & 33,3 & 2,8 & 70,8 \\
\hline & & F12 & 100,0 & 76,2 & 61,9 & 57,1 & 4,3 & 117,3 \\
\hline & & Média & 91,1 & 51,0 & 44,2 & 42,8 & 3,2 & 90,1 \\
\hline
\end{tabular}

R. Árvore, Viçosa-MG, v.33, n.5, p.811-819, 2009 
Tabela 2-Análise de deviance para proporção de pegamentos em esquema fatorial cruzado a partir de sete enxertos de Ilex paraguariensis de diferentes matrizes e posições durante o período de acompanhamento.

Table 2 - Deviance analysis for survivals proportion in outline crossed factorial based on seven grafts of Ilex paraguariensis of different mother trees and positions over the period of study.

\begin{tabular}{|c|c|c|c|c|c|c|}
\hline \multirow[t]{2}{*}{ Fonte de variação } & \multicolumn{3}{|c|}{ Matrizes > 80 anos } & \multicolumn{3}{|c|}{ Matrizes 10 anos } \\
\hline & Deviance ${ }^{2}$ & Graus de liberdade & p-valor ${ }^{1}$ & Deviance ${ }^{2}$ & Graus de liberdade & p-valor ${ }^{1}$ \\
\hline Matriz & 727.44 & 6 & $<0.001$ & 690.12 & 6 & $<0.001$ \\
\hline Posição & 721.87 & 2 & 0.0618 & 685.21 & 2 & 0.0856 \\
\hline Período & 590.80 & 3 & $<0.001$ & 609.23 & 3 & $<0.001$ \\
\hline Matriz * Período & 497.79 & 18 & 0.5984 & 546.06 & 18 & 0.0130 \\
\hline Matriz * Posição & 513.70 & 12 & $<0.001$ & 579.95 & 12 & 0.0036 \\
\hline Posição * Período & 491.53 & 6 & 0.3957 & 539.88 & 6 & 0.4033 \\
\hline Resíduo & 806.95 & 508 & & 732.36 & 504 & \\
\hline
\end{tabular}

${ }^{1}$ : nível de probabilidade pelo teste qui-quadrado; ${ }^{2}$ Distribuição binomial, ligação Logit.

A análise de modelos lineares mistos evidenciou comportamento diferenciado das matrizes quando amostrados em diferentes posições de coleta. De acordo com essas evidências, há a possibilidade de identificar os efeitos fixos de posição que melhor atendam ao processo de enxertia de Ilex paraguariensis. O efeito não significativo da interação Posição de coleta e Período de acompanhamento faz que o modelo ajustado seja melhor que, quando observado ao longo do tempo, de forma que a escolha da posição de coleta, para a resposta de pegamento dos enxertos possa ser antecipada, ou seja, períodos inferiores a 180 dias. Dessa forma, optou-se pela avaliação genética por período, de forma independente.

A análise de deviance, para os caracteres número de brotos e comprimento de brotos aos 180 dias após a enxertia (Tabelas 3 e 4), mostrou efeito significativo (valor $\mathrm{p}<0,0001)$ apenas para o efeito de matriz para a população de 10 anos. O efeito da interação Matriz*Posição de coleta somente foi evidenciado para o caráter comprimento de brotos nas matrizes obtidas das matrizes superiores a 80 anos. Evidencia-se, assim, que as posições de coleta devem ser observadas, com maior rigor, quando as matrizes de onde será retirado o material propagativo (enxerto) se encontram em idade avançada.

A variabilidade genética entre as matrizes pode ser observada pelo coeficiente de variação genético e pela estimativa de herdabilidade. Através do desvio padrão da herdabilidade, que foi de pequena magnitude, pode-se garantir que esta não é nula, evidenciando, assim, a variabilidade genética e a possibilidade de ganho com a seleção (Tabela 5).

A herdabilidade individual dos efeitos genotípicos, estimada para o pegamento dos enxertos, foi superior a $74 \%$ para todos os períodos de avaliação, cujo valor é considerado alto. Isto indica que grande parte da variabilidade fenotípica desse caráter foi devida a variação genética das matrizes de Ilex paraguariensis. Ressalta-se que não foram detectadas diferenças significativas entre porta-enxertos. Desse modo, a seleção individual pode ser eficiente, ou seja, ao selecionarem-se matrizes com base em seus valores genéticos preditos, empregando os valores individuais; espera-se aumentar a proporção de pegamentos dos enxertos da espécie.

Tabela 3 - Análise de deviance para número de brotos aos 180 dias em esquema fatorial cruzado a partir de sete enxertos de Ilex paraguariensis de diferentes matrizes e posições.

Table 3 - Deviance analysis for number of sprouts at 180 days in outline crossed factorial based on seven grafts of Ilex paraguariensis of different mother trees and positions.

\begin{tabular}{|c|c|c|c|c|c|c|}
\hline \multirow[t]{2}{*}{ Fonte de variação } & \multicolumn{3}{|c|}{ Matrizes $>80$ anos } & \multicolumn{3}{|c|}{ Matrizes 10 anos } \\
\hline & Deviance ${ }^{2}$ & Graus de liberdade & p-valor ${ }^{1}$ & Deviance 2 & Graus de liberdade & p-valor ${ }^{1}$ \\
\hline Matriz & 43.56 & 6 & 0.0661 & 78.81 & 6 & $<0.0001$ \\
\hline Posição & 43.27 & 2 & 0.8312 & 73.42 & 2 & 0.0672 \\
\hline Matriz * Posição & 34.17 & 9 & 0.2207 & 63.28 & 11 & 0.5178 \\
\hline Resíduo & 52.62 & 47 & & 110.06 & 62 & \\
\hline
\end{tabular}

${ }^{1}$ Nível de probabilidade pelo teste qui-quadrado; ${ }^{2}$ Distribuição de Poisson, Ligação Log. 
Tabela 4 -Análise de deviance para comprimento de brotos aos 180 dias em esquema fatorial cruzado a partir de sete enxertos de Ilex paraguariensis de diferentes matrizes e posições.

Table 4 - Deviance analysis for sprouts length at 180 days in outline crossed factorial based on seven grafts of Ilex paraguariensis of different mother trees and positions.

\begin{tabular}{|c|c|c|c|c|c|c|}
\hline \multirow[t]{2}{*}{ Fonte de variação } & \multicolumn{3}{|c|}{ Matrizes $>80$ anos } & \multicolumn{3}{|c|}{ Matrizes 10 anos } \\
\hline & Deviance ${ }^{2}$ & Graus de liberdade & p-valor ${ }^{1}$ & Deviance ${ }^{2}$ & Graus de liberdade & p-valor ${ }^{1}$ \\
\hline Matriz & 52.26 & 6 & 0.2591 & 63.66 & 6 & $<0.0001$ \\
\hline Posição & 51.99 & 2 & 0.7470 & 62.04 & 2 & 0.2517 \\
\hline Matriz * Posição & 40.34 & 9 & 0.0027 & 52.56 & 11 & 0.1349 \\
\hline Resíduo & 55,82 & 47 & & 87,38 & 62 & \\
\hline
\end{tabular}

${ }^{1}$ Nível de probabilidade pelo teste qui-quadrado; ${ }^{2}$ Distribuição Gama, ligação Log.

Tabela 5 - Estimativas de parâmetros genéticos para o pegamento e vigor de enxertos de Ilex paraguariensis. Table 5 - Estimates of genetic parameters for the survival and vigor of grafts of Ilex paraguariensis.

\begin{tabular}{|c|c|c|c|c|c|c|}
\hline \multirow[b]{2}{*}{ Parâmetros } & \multicolumn{4}{|c|}{ Pegamento } & \multicolumn{2}{|c|}{ Vigor aos 180 dias } \\
\hline & 30 dias & 90 dias & 120 dias & 180 dias & Nbrotos & Cbrotos \\
\hline Variância genotípica entre matrizes & 0.009606 & 0.040627 & 0.032109 & 0.029854 & 0.7880 & 665.35 \\
\hline Variância residual (ambiental) & 0.062118 & 0.205211 & 0.219683 & 0.222303 & 3.46 & 3769.04 \\
\hline Variância fenot. individual & 0.071724 & 0.245838 & 0.251792 & 0.252157 & 4.24 & 4434.39 \\
\hline $\begin{array}{l}\text { Herdabilidade individual } \\
\text { dos efeitos genotípicos }\end{array}$ & $0.765 \pm 0.0604$ & $0.806 \pm 0.0671$ & $0.755 \pm 0.0589$ & $0.737 \pm 0.0568$ & $0,406 \pm 0,0661$ & $0,346 \pm 0,0713$ \\
\hline Acurácia da seleção de matrizes & 0.562936 & 0.610427 & 0.552107 & 0.535893 & 0.6373 & 0.5884 \\
\hline Coeficiente de variação genético (\%) & 10.64187 & 34.64077 & 34.63232 & 34.53155 & 25.36 & 30.51 \\
\hline Coeficiente de variação experimental & 27.06218 & 77.85368 & 90.58709 & 94.22974 & 53.10 & 72.62 \\
\hline
\end{tabular}

O coeficiente de variação genética foi da ordem de $34 \%$, nos períodos de acompanhamento iguais ou superiores a 90 dias, indicando boas perspectivas de ganho no melhoramento do material genético. $\mathrm{O}$ coeficiente de variação experimental, nesse mesmo período, ficou entre $78 \%$ e $94 \%$, considerado de alta magnitude, porém foi coerente com os valores obtidos para o caráter avaliado em condições normais de campo.

Pode-se observar, à exceção do coeficiente de variação experimental, que não houve diferenças entre os parâmetros genéticos a partir dos 90 dias, indicando a possibilidade de avaliação do pegamento dos enxertos aos 90 dias. Isto pode ser comprovado pelos ordenamentos das matrizes nesses períodos (Tabela 6). Em termos de um programa com enxertia da espécie, isso se torna particularmente importante, em vista de uma avaliação de sobrevivência aos 90 dias após a enxertia ser suficiente para a obtenção de estimativas confiáveis, não necessitando mais avaliações.

Para a variável pegamento, a matriz A3 ocupou a primeira ordem no ranking efetuado para valores genéticos preditos. Destaca-se que sete matrizes, aos 90 dias, tiveram valores preditos positivos e mantiveram essa classificação (positivo) nas avaliações de 120 e 180 dias. Assim, pode-se inferir que essas matrizes proporcionarão ganhos, em relação ao caráter pegamento, com a seleção, além da visível influência da matriz no pegamento dos enxertos.

Conforme a Tabela 7 e baseando na avaliação aos 90 dias, percebe-se, de maneira geral, que os enxertos provenientes de matrizes com 10 anos de idade apresentaram pegamento superior àqueles com idades acima de 80 anos, uma vez que possuem mais representantes com efeito genotípico predito positivo. Segundo Boliani (1986), o estádio juvenil possibilita maior crescimento vegetativo da planta e a produção de grande área foliar, como também a produção de fotoassimilados a serem, posteriormente, utilizados no desenvolvimento dos frutos e do sistema radicular, facilitando a absorção de água e íons do solo. Baseando-se nisso, e em função dos resultados obtidos neste estudo, pode-se inferir que a enxertia apresenta maiores chances de sucesso, em termos de pegamento, quando se usa material proveniente de plantas mais novas como enxerto.

Em relação ao comprimento e número de brotos dos enxertos, percebe-se um comportamento distinto daquele apresentado para o pegamento dos enxertos, sem uma tendência claramente visível. 
Tabela 6-Efeitos genotípicos preditos para o pegamento, comprimento e número de brotos de enxertos de Ilex paraguariensis. Table 6 - Predicted genetics effects for the survival, length and number of sprouts of grafts of Ilex paraguariensis.

\begin{tabular}{|c|c|c|c|c|c|c|c|c|c|c|c|}
\hline \multicolumn{8}{|c|}{ Pegamentos } & \multicolumn{2}{|c|}{ Cbrotos } & \multicolumn{2}{|c|}{ Nbrotos } \\
\hline \multicolumn{2}{|c|}{30 dias } & \multicolumn{2}{|c|}{90 dias } & \multicolumn{2}{|c|}{120 dias } & \multicolumn{2}{|c|}{180 dias } & \multirow[b]{2}{*}{ Matriz } & \multirow[b]{2}{*}{ G } & \multirow[b]{2}{*}{ Matriz } & \multirow[b]{2}{*}{ G } \\
\hline Matriz & $\mathrm{G}$ & Matriz & $\mathrm{G}$ & Matriz & $\mathrm{G}$ & Matriz & $\mathrm{G}$ & & & & \\
\hline A 1 & 0.0604 & A 3 & 0.2219 & A 3 & 0.1485 & A3 & 0.1579 & A 2 & 34.38 & A 8 & 1.32 \\
\hline A3 & 0.0604 & F2 & 0.1559 & A9 & 0.1485 & A 10 & 0.1479 & F8 & 23.80 & A 2 & 1.03 \\
\hline A9 & 0.0604 & A9 & 0.1451 & A 10 & 0.1387 & F2 & 0.1365 & A 8 & 22.71 & F8 & 0.75 \\
\hline M1 & 0.0604 & M1 & 0.1451 & F2 & 0.1266 & A9 & 0.1228 & M1 & 20.34 & M1 & 0.51 \\
\hline F8 & 0.0604 & A 10 & 0.137 & A 7 & 0.0767 & A 2 & 0.0525 & $\mathrm{~F} 4$ & 8.48 & $\mathrm{~F} 2$ & 0.50 \\
\hline $\mathrm{F} 2$ & 0.059 & A 8 & 0.1067 & A 8 & 0.0767 & A7 & 0.0525 & F7 & 0.02 & F4 & 0.03 \\
\hline A 2 & 0.024 & A7 & 0.0684 & M1 & 0.0767 & A 8 & 0.0525 & $\mathrm{~F} 2$ & -0.03 & F7 & -0.06 \\
\hline A7 & 0.024 & A 2 & -0.0084 & $\mathrm{~A} 2$ & 0.0407 & M1 & 0.0525 & F1 & -2.81 & A7 & -0.22 \\
\hline $\mathrm{F} 1$ & 0.024 & F7 & -0.0084 & F7 & 0.0048 & F7 & 0.0173 & A 10 & -7.09 & A 10 & -0.30 \\
\hline F3 & 0.024 & F1 & -0.0468 & F3 & 0.0048 & F3 & 0.0173 & A 7 & -0.1099 & F3 & -0.48 \\
\hline F7 & -0.0488 & F3 & -0.0468 & F1 & -0.1029 & F1 & -0.1233 & A 1 & -12.07 & F1 & -0.61 \\
\hline A 8 & -0.0852 & F8 & -0.2003 & F8 & -0.1388 & F8 & -0.1233 & F3 & -17.03 & A3 & -0.72 \\
\hline A 10 & -0.0923 & A 1 & -0.2771 & A 1 & -0.2466 & A 1 & -0.2287 & A 3 & -21.42 & A1 & -0.72 \\
\hline F4 & -0.2308 & F4 & -0.3922 & F4 & -0.3543 & $\mathrm{~F} 4$ & -0.3342 & A9 & -38.31 & A9 & -1.04 \\
\hline
\end{tabular}

G: é o efeito genotípico predito.

Tabela 7 -Média dos efeitos fixos para a posição de coleta de enxertos dentro das plantas matrizes de Ilex paraguariensis em relação ao pegamento, comprimento de brotos (Cbrotos) e número de brotos (Nbrotos) dos enxertos.

Table 7 - Average fixed effects for the position of collection of grafts inside of the mother tree of Ilex paraguariensis in relation to the survival, length of sprouts (Cbrotos) and number of sprouts (Nbrotos) of the grafts.

\begin{tabular}{|c|c|c|c|c|c|c|c|}
\hline \multirow[t]{2}{*}{ Posição } & \multirow[t]{2}{*}{ Idade das matrizes } & \multicolumn{4}{|c|}{ Pegamento dos enxertos } & \multirow{2}{*}{$\begin{array}{l}\text { Cbrotos } \\
180 \text { dias }\end{array}$} & \multirow{2}{*}{$\begin{array}{l}\text { Nbrotos } \\
180 \text { dias }\end{array}$} \\
\hline & & 30 dias & 90 dias & 120 dias & 180 dias & & \\
\hline \multirow[t]{2}{*}{ Ápice } & $>$ 80anos & 0.96 & 0.50 & 0.40 & 0.38 & 31.69 & 1.39 \\
\hline & 10anos & 1.00 & 0.65 & 0.59 & 0.59 & 41.89 & 1.83 \\
\hline \multirow[t]{2}{*}{ Base } & $>80$ anos & 0.87 & 0.52 & 0.47 & 0.47 & 44.35 & 1.80 \\
\hline & 10anos & 0.92 & 0.59 & 0.53 & 0.49 & 44.42 & 1.87 \\
\hline \multirow[t]{2}{*}{ Meio } & $>80$ anos & 0.91 & 0.59 & 0.52 & 0.50 & 42.68 & 1.78 \\
\hline & 10anos & 0.87 & 0.71 & 0.65 & 0.63 & 43.96 & 1.92 \\
\hline
\end{tabular}

A média dos efeitos fixos estimados para a posição de coleta dos enxertos mostrou-se significativa $(\mathrm{p}$-valor $=0,0171)$. De acordo com os valores observados, a posição apical proporcionou a melhor resposta ao pegamento dos enxertos (Tabela 7). Entre os fatores que afetam a propagação por enxertia, segundo Rocha et al. (2002), estão as condições do propágulo, ou seja, o estádio de maturação da planta doadora, a posição do propágulo na planta-matriz e o tipo de propágulo utilizado.

Em estudos com Pinus taeda e Pinus elliottii, Filho (1970) estudou a influência da posição dos ramos na copa sobre o pegamento dos enxertos. Segundo esse autor, para Pinus taeda a maior taxa de pegamento foi de ramos retirados do terço médio da copa $(90 \%)$ e a menor sobrevivência em ramos retirados do terço inferior da copa (20\%). Para o $P$. elliottii, não houve grande variação na porcentagem de sobrevivência, com relação à posição da qual foram coletados os ramos.

Em termos de enxertia de erva-mate, não se têm resultados de literatura buscando avaliar os efeitos do local de coleta dos enxertos na planta-matriz. Já na estaquia, diversas pesquisas procurando estabelecer esse efeito foram desenvolvidas. Prat Krikum et al. (1983), obtiveram $50,6 \%$ e $48,6 \%$ de enraizamento, respectivamente, de propágulos oriundos das partes mediana e basal, em comparação com 21,2\% da parte apical, sendo a mesma tendência encontrada por Prak Krikun (1986). Graça et al. (1988) concluíram que, na estaquia a partir de mudas oriundas de sementes e mantidas em casa de vegetação, podem ser utilizadas estacas de quaisquer seções dos ramos, embora haja tendência de maior sucesso com estacas nas seções basal e mediana. Para Tavares et

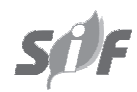

R. Árvore, Viçosa-MG, v.33, n.5, p.811-819, 2009 
al. (1992), estacas coletadas das seções mediana e basal apresentaram maior enraizamento do que as retiradas da seção apical.

Baseando no conceito de juvenilidade e maturidade dos propágulos (GREENWOOD e HUTCHISON, 1993), no presente estudo esperava-se maior taxa de pegamento dos enxertos provenientes de brotações da base. Entretanto, em função de brotações do ápice, em geral, serem menos lignificadas, esse efeito pode ter sido predominante em relação à posição dos propágulos na planta-matriz. Aliado a isso, no caso das matrizes de erva-mate utilizadas neste estudo a poda bi-anual para coleta de folhas e ramos pode ter mascarado a influência da idade ontogenética dos propágulos sobre o pegamento dos enxertos.

No caso da erva-mate, o método de enxertia ora apresentado, é recomendado para a implantação de pomares de produção de sementes, bancos clonais e para o resgate e rejuvenescimento de propágulos adultos, visando ao seu enraizamento futuro pela técnica de estaquia. A implantação de áreas para produção comercial de ervamate com a técnica da sobre-enxertia ainda precisa ser investigada quanto à produtividade e longevidade das plantas obtidas. No caso da Hevea brasiliensis, a enxertia é a técnica recomendada para o estabelecimento de plantios clonais (MEDRADO, 1992; SOUZA et al., 1990).

A sobre-enxertia de Ilex paraguariensis sobre brotações de plantas adultas decepadas, visando à substituição da parte aérea por genótipos de interesse, é uma técnica de extrema importância para a implementação da silvicultura clonal da espécie. Sendo a Ilex paraguariensis espécie perene, além da grande quantidade de ervais já implantados sem os devidos cuidados com a seleção de material genético para características de interesse específicas (sabor, crescimento e resistência a doenças), o método de enxertia desenvolvido e ora apresentado, possibilita a troca e padronização da qualidade do produto a ser obtido, sem a necessidade da substituição dos ervais já implantados, o que resultará em ganhos aos produtores.

\section{CONCLUSÕES}

Com base nos resultados deste estudo, pode-se concluir que:

1. A enxertia de erva-mate sobre brotações oriundas de árvores adultas decepadas diretamente a campo é tecnicamente viável.

R. Árvore, Viçosa-MG, v.33, n.5, p.811-819, 2009
2. Tanto a idade das plantas-matriz quanto o local de coleta dos enxertos dentro delas são importantes no pegamento da enxertia, apresentando estes melhores resultados para árvores mais novas e com brotações apicais.

3. Os efeitos de porta-enxerto foram não significativos e as matrizes apresentam respostas diferenciadas quanto ao pegamento na enxertia.

4. A avaliação do pegamento feita aos 90 dias após a enxertia se mostrou eficiente na avaliação da técnica.

\section{AGRADECIMENTOS}

Os autores agradecem a Empresa Baldo S.A. e ao Dr. José Alfredo Sturion (Embrapa Florestas) pela seleção e disponibilização do material genético utilizado nos estudos.

\section{REFERÊNCIAS}

BOLIANI, A. C. Efeitos do estiolamento basal, da juvenilidade e do uso de um regulador vegetal no enraizamento de estacas de raízes e de ramos herbáceos de algumas espécies frutíferas. 1986. 129f.

Dissertação (Mestrado em Agronomia) - Escola Superior de Agricultura “Luiz de Queiroz”, Piracicaba, 1986.

BRAGAGNOLO, N.; PAN, W.; KLOSOVSKI

FILHO, L. Manual técnico de erva-mate. Curitiba: EMATER-PR, 1980. 40p.

COLLETT, D. Modeling binary data.

London: Chapman and Hall, 1991. 369p.

EMPRESA BRASILEIRA DE PESQUISA AGROPECUÁRIA - EMBRAPA. Enxertia de copa em seringueira. Manaus: 1989. 148p. (Documentos, 7)

GRAÇA, M. E. C. et al. Estaquia de erva-mate. Colombo: Embrapa, 1988. 6p. (Circular Técnica, 18)

GREENWOOD, M. S.; HUTCHISON, K. W. Maturation as an developmental process. In: AHUJA, M. R.; LIBBY, W. J. Clonal forestry: genetics and biotechnology. Budapest: Springer-Verlag, 1993. p.14-33.

LITTEL, R. C.; MILLIKEN, G. A.; STROUP, W. W. SAS system for mixed models. Cary: SAS Institute, 1996. 633p. 
MACCARI JÚNIOR. A.; MAZUCHOWSKI, J.Z. Produtos alternativos e desenvolvimento da tecnologia industrial na cadeia produtiva da erva-mate. Curitiba: Câmara Setorial Produtiva da Erva-Mate do Paraná, 2000. 160p.

MEDRADO, M. J. S. Fatores relacionados ao processo de propagação da seringueira (Hevea spp), em Piracicaba, SP. 1992. 178f. Tese (Doutorado em Agronomia) Escola Superior de Agricultura “Luiz de Queiroz”, Piracicaba, 1992.

MOOD, A. M.; GRAYBILL, F. A.; BOES, D. C. Introduction to the theory of statistics. Tokyo: McGraw-Hill, 1974. 564p.

PINHEIRO, E. et al. A enxertia de copa na formação de seringais de cultivo nos trópicos úmidos da Amazônia. Belém: FCAP, 1988. 27p. (Informe Técnico, 13)

PRAT KRIKUN, S.D. et al. Yerba mate. Informe sobre investigaciones realizadas. Período 1984-85. Cerro Azul: E.E.A. Misiones, 1986. 32p.(Publicación Miscelânea, 15)

PRAT KRIKUN, S. D. et al. Yerba mate. Informe sobre investigaciones realizadas. Período 1982-83. Cerro Azul: E.E.A. Misiones, 1983. 32p. (Publicación Miscelânea, 7)

RESENDE, M. D. V. Genética biométrica e estatística no melhoramento de plantas perenes. Brasília, DF: Embrapa Informação Tecnológica, 2002.975p.

RESENDE, M. D. V. Software selegenREML/BLUP. Colombo: Embrapa, 2002. 67p (Documentos 77)
ROCHA, M. G. B. et al. Propagação vegetativa de espécies arbóreas nativas. In. ROCHA, M. G. B.

Melhoramento de espécies arbóreas nativas. Belo Horizonte: Instituto Estadual de Florestas, 2002.p.91-108.

SOUZA, L. H. et al. Armazenamento de hastes de seringueira (Hevea brasiliensis) "clone RRIM 600". I. Efeito dos métodos de armazenamento sobre o índice de pegamento da enxertia.

Revista Árvore, v.14, n.1, p.69-77, 1990.

Sturion, J. A. Produção de mudas e implantação de povoamentos com erva-mate. Curitiba, Embrapa-CNPF, 1988. 10p. (Circular Técnica, 17)

SUITER FILHO, W. S. Influência da posição do ramo da copa na enxertia de Pinus elliottii Engel. e Pinus taeda L. IPEF, v.1, p.121-124, 1970.

TAVARES, F. R.; PICHET, J. A.; MASCHIO, L. M. A. Alguns fatores relacionados com a estaquia da erva-mate (Ilex paraguariensis St. Hil.). In: CONGRESSO FLORESTAL ESTADUAL, 7, 1992, Santa Maria. Anais... Santa Maria: UFSM, 1992. p.626-640.

WENDLING, I.; SOUZA JÚNIOR, L. Propagação vegetativa de erva-mate (Ilex paraguariensis Saint Hilaire) por miniestaquia de material juvenil. In: CONGRESSO SUL-AMERICANO DAERVAMATE, 3., 2003, Chapecó. Anais... Chapecó: EPAGRI, 2003.

XAVIER, A. et al. Desempenho do enraizamento de microestacas e miniestacas de clones de híbrido de Eucalyptus grandis. Revista árvore, v.25, n.4, p.403-411, 2001. 